$\underline{\text { Supporting Information }}$

\title{
Controlling the Resistive Switching Behavior in Starch-based Flexible Biomemristors
}

Niloufar Raeis-Hosseini and Jang-Sik Lee*

N. Raeis-Hosseini, Prof. J. -S. Lee

Department of Materials Science and Engineering, Pohang University of Science and

Technology (POSTECH), Pohang, 790-784, South Korea

*E-mail: jangsik@postech.ac.kr 


\section{Table of the Contents}

\section{Schematics}

1. Schematic formula of starch and chitosan........................................ 1

2. Interaction between starch and chitosan........................................ 2

\section{Figures}

3. Cross-sectional SEM images................................................ 1

4. $I-V$ characteristics with different compliance currents.......................... 2

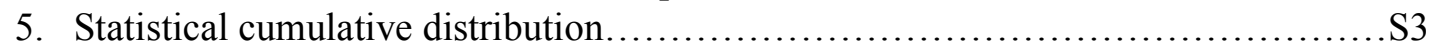

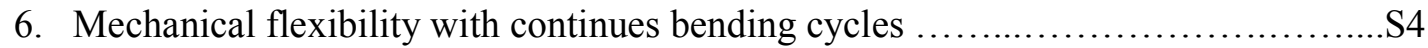

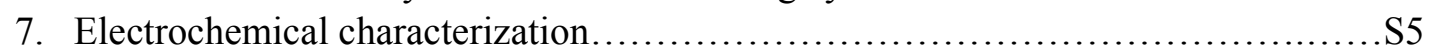

8. Effect of different electrodes on RS properties................................ 6

9. Effect of thickness on RS properties for both of the bio-ReRAMs .................S7

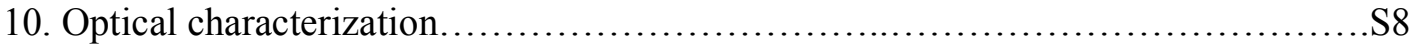


(a)

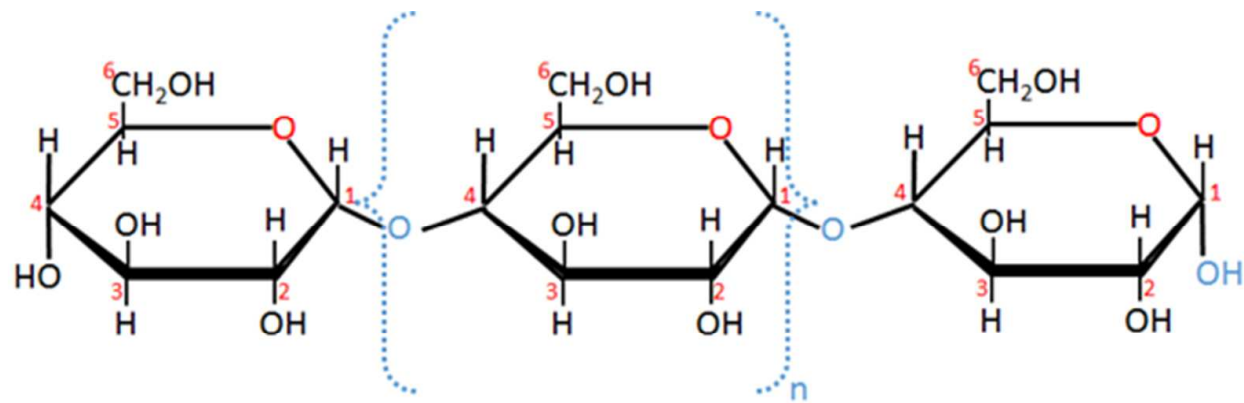

Amylose: $\alpha(1-4)$ - glycosidic linkage

(b)
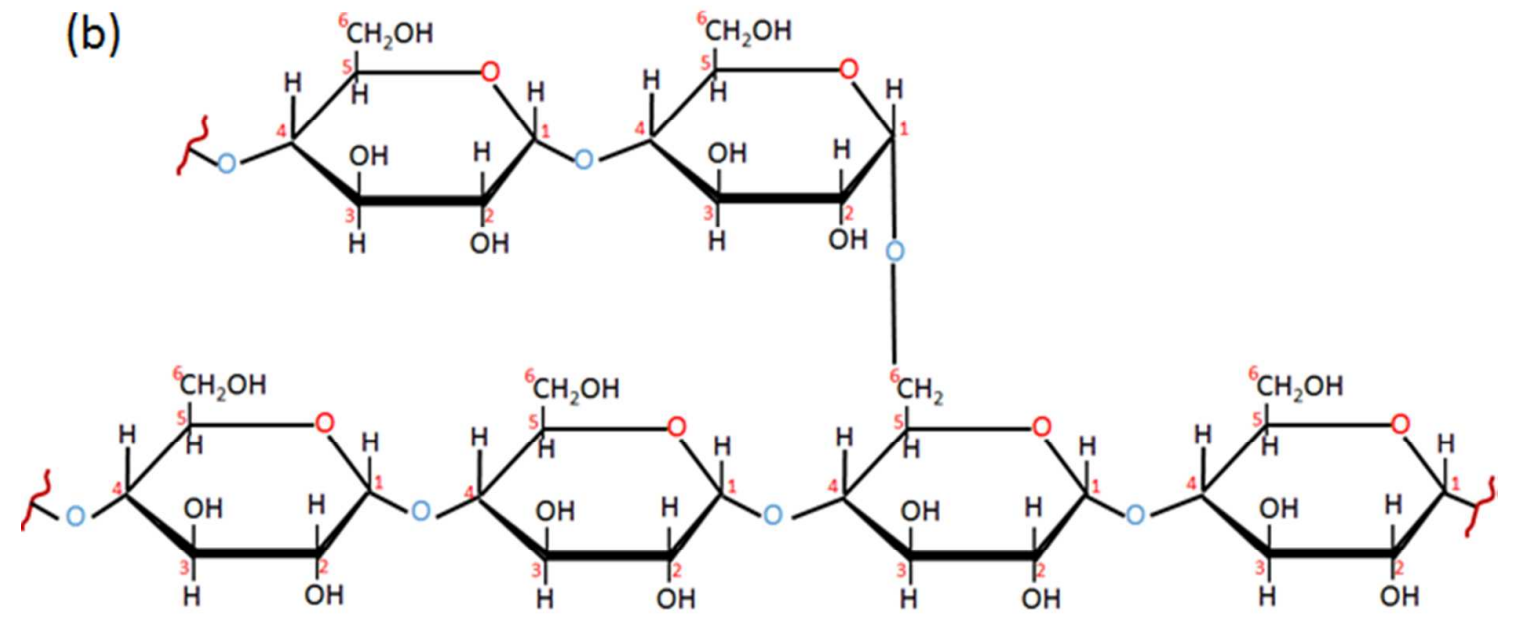

Amylopectin: $\alpha(1-6)$ - glycosidic linkage

(c)

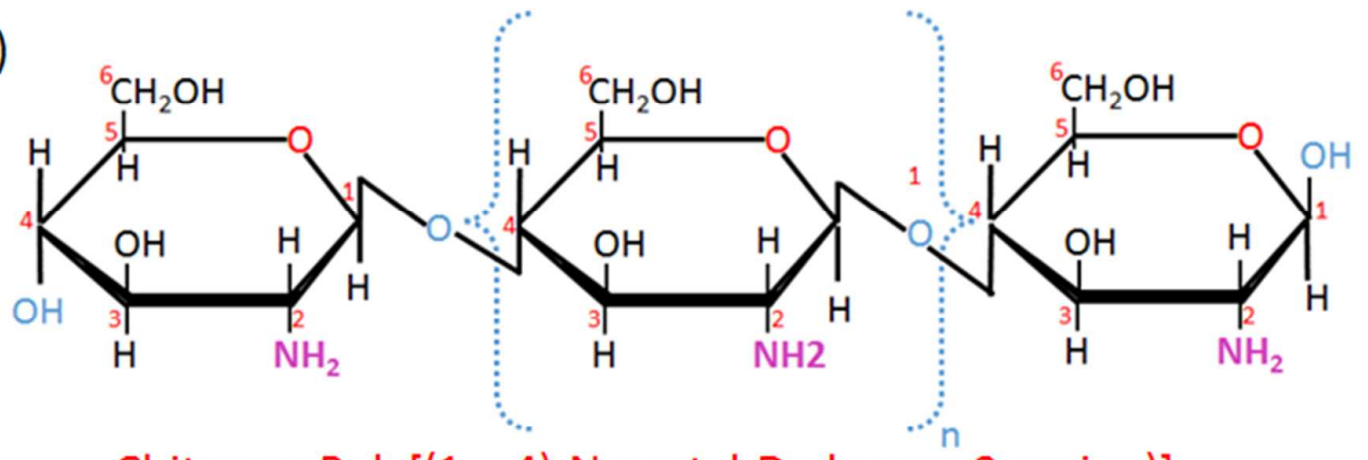

Chitosan: Poly[(1 - 4)-N-acetyl-D-glucose-2-amine $)]$

Supporting Schematic S1. Illustration of the schematic formula of (a) amylose, (b) amylopectin, and (c) chitosan. 


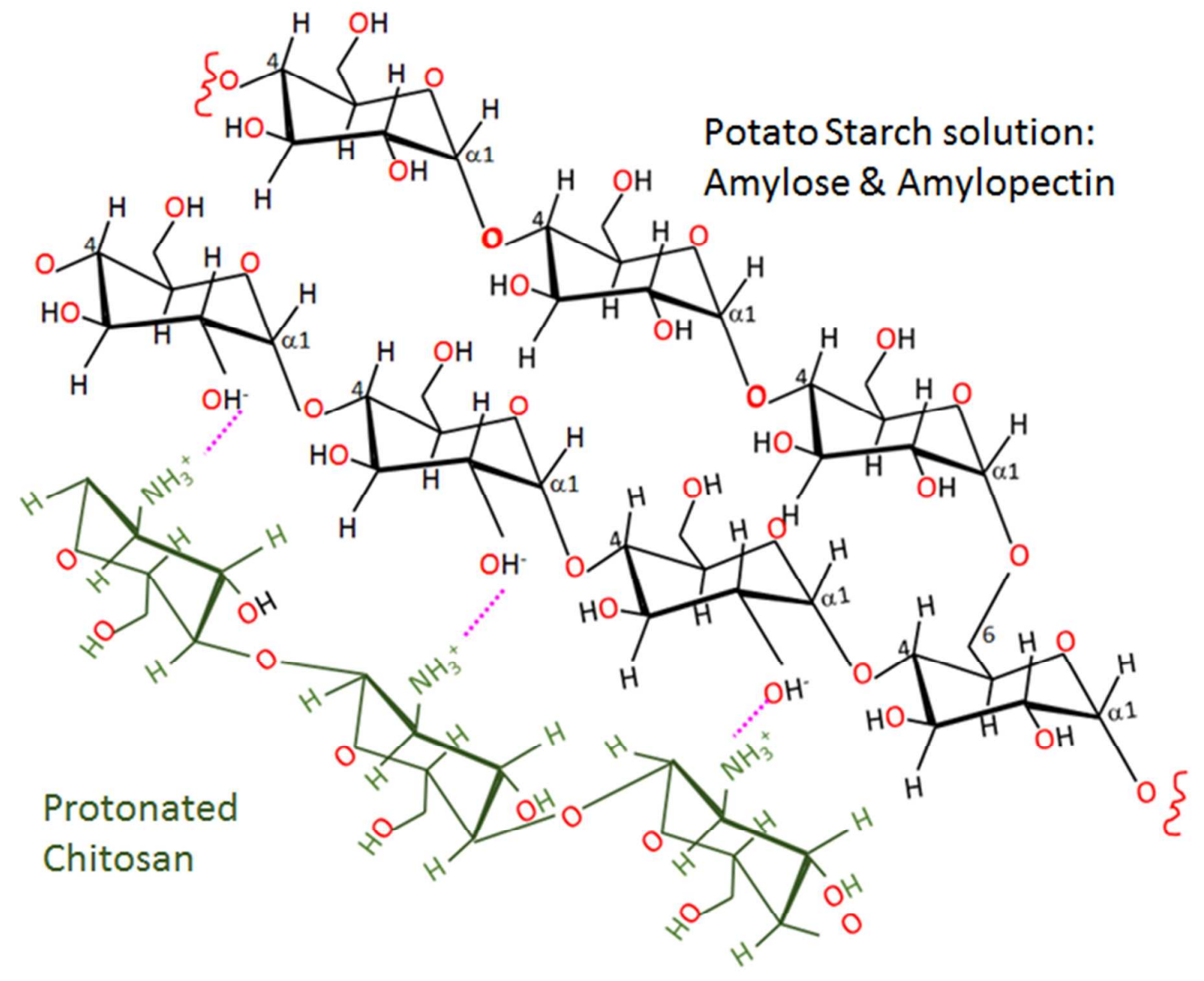

Supporting Schematic S2. Postulated interaction between starch and chitosan with blending two bioinspired polymers. 
(a)

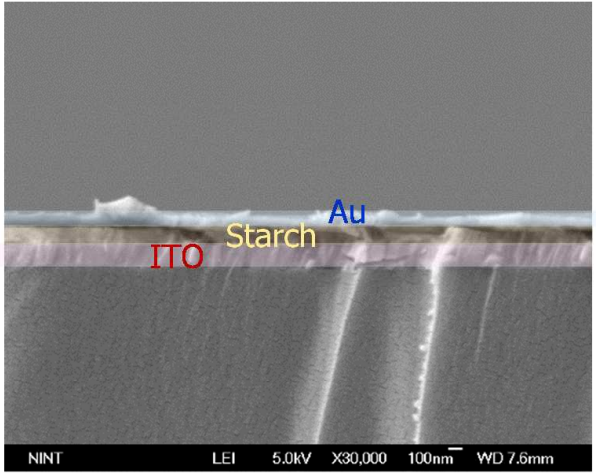

(b)

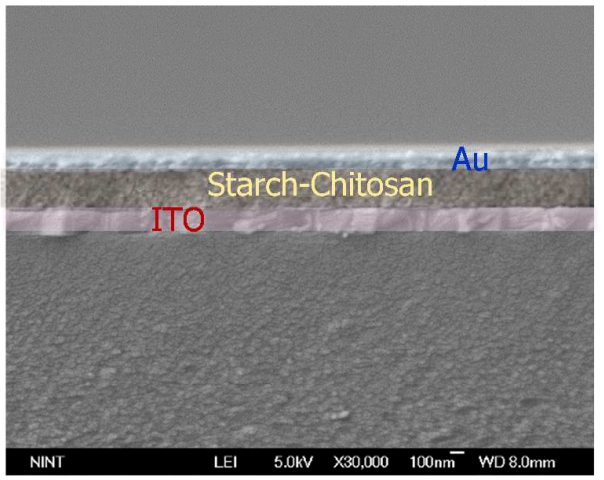

Supporting Figure S1. Cross-sectional SEM image for devices with (a) Au/starch /ITO/PET, and (b) Au/starch-chitosan/ITO/PET structures. 

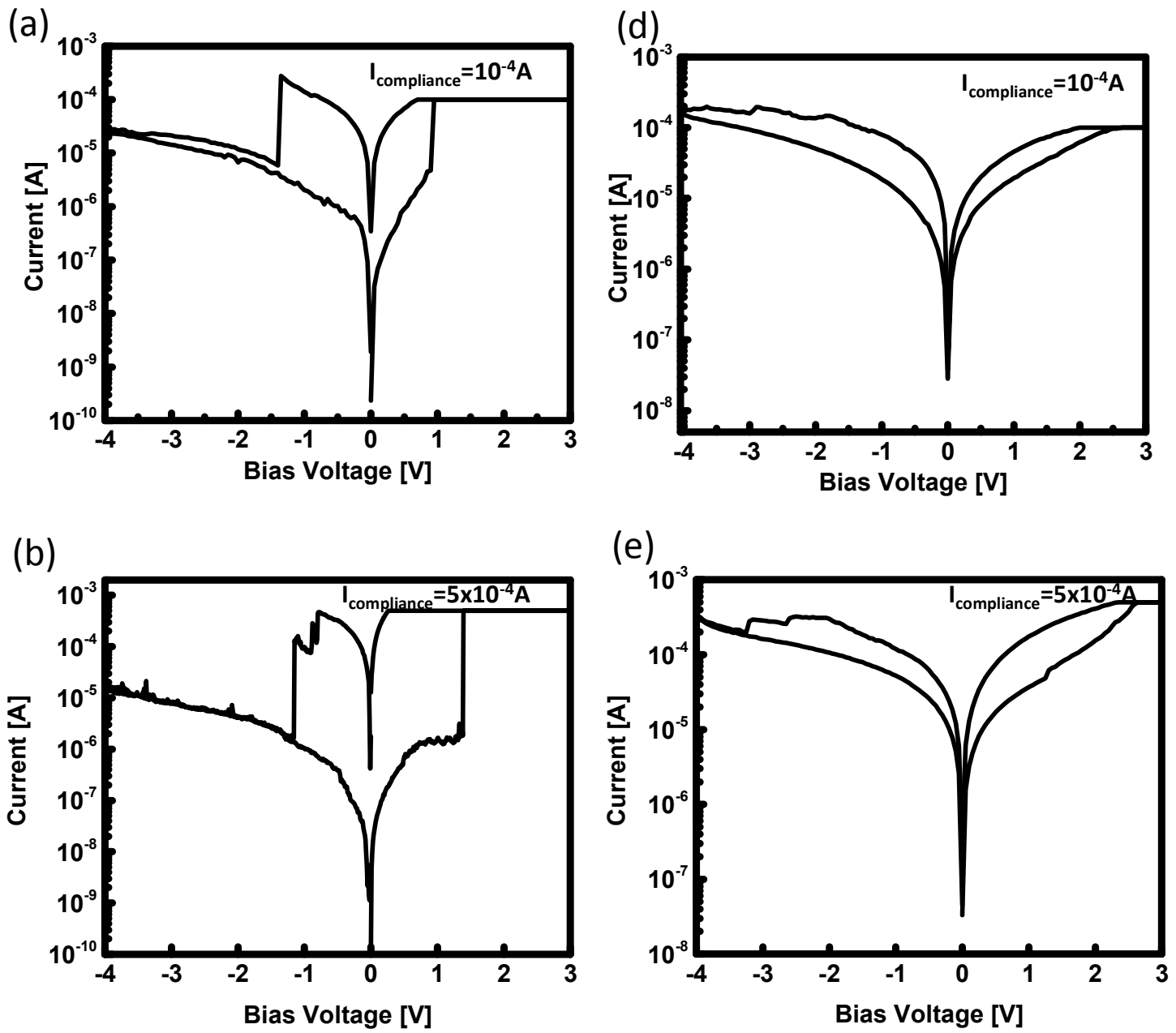

(c)

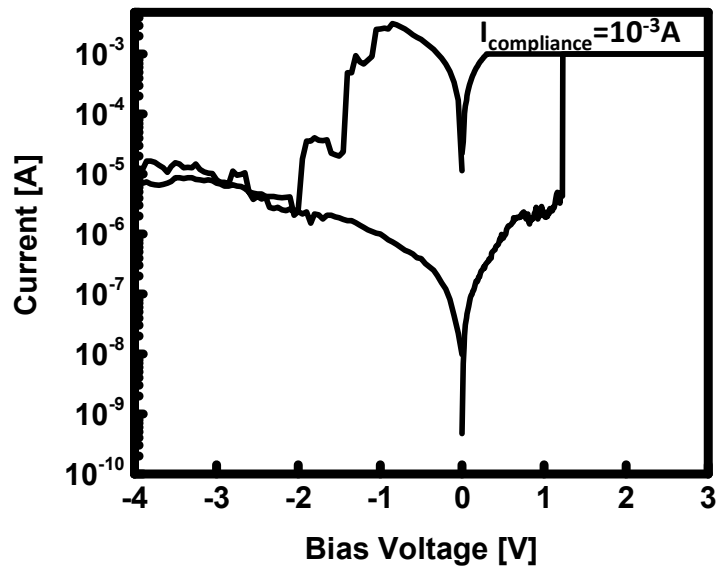

(f)

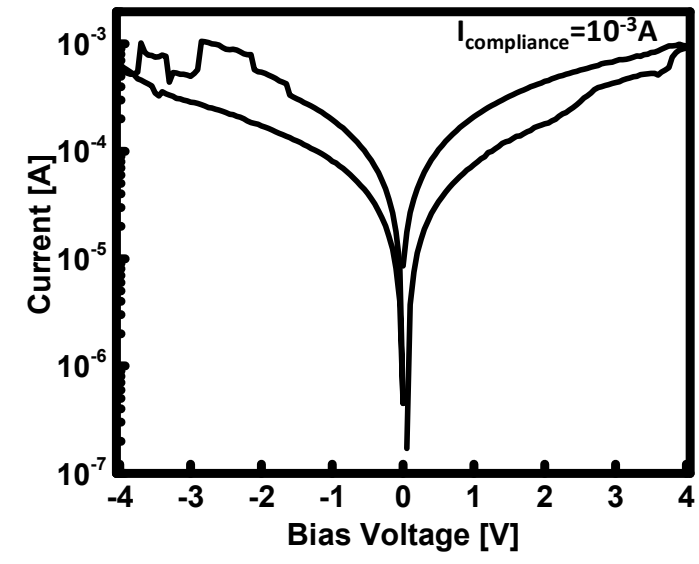

Supporting Figure S2. ) I- $V$ characteristics with different compliance currents for (a-c) starch-based devices and (d-f) starch-chitosan-based bio-ReRAMs. 


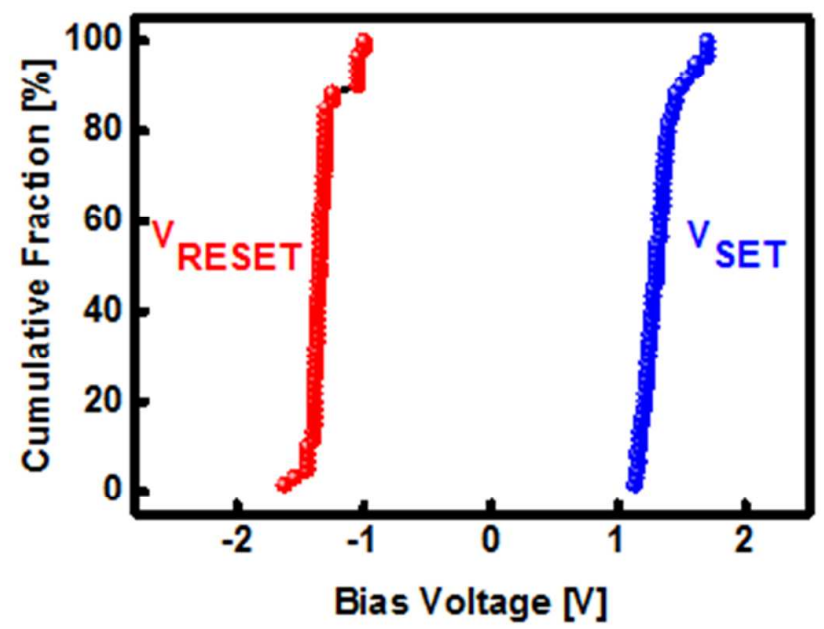

Supporting Figure S3. Cycle-to-cycle statistical cumulative distribution of the SET and RESET voltages for devices with Au/starch /ITO/PET structure up to 60 cycles. 

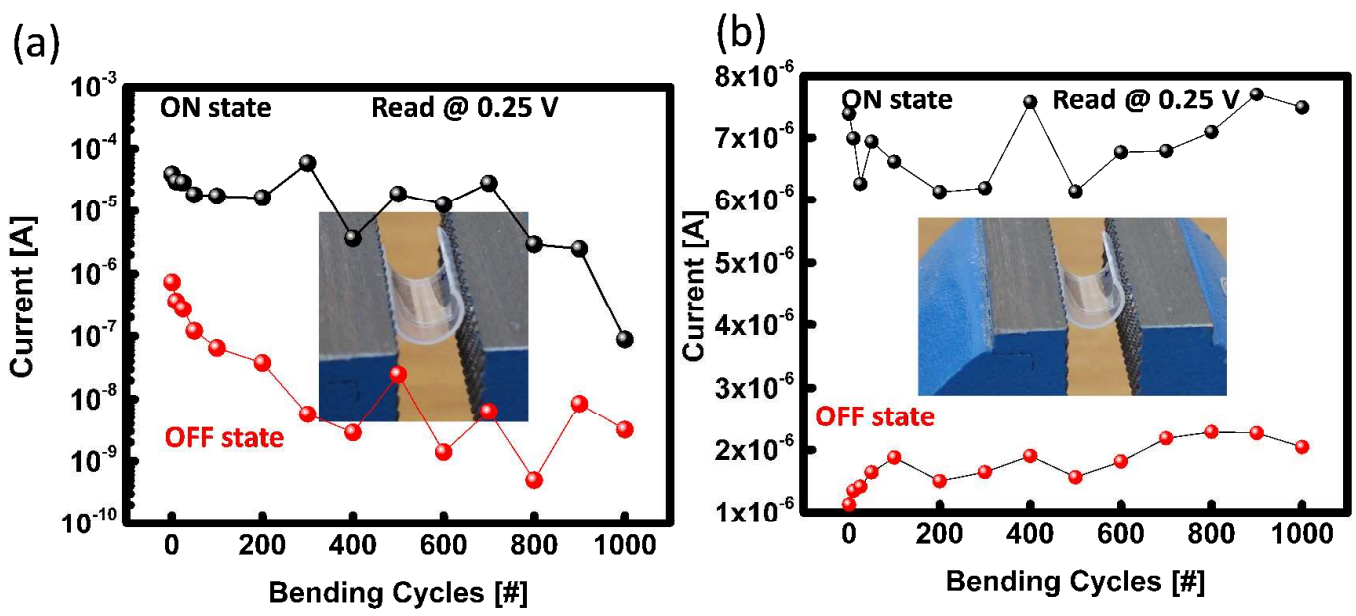

Supporting Figure S4. ON and OFF current states of (a) starch-based and (b) starchchitosan-based devices as a function of bending cycles while the curvature radius of $5 \mathrm{~mm}$ is utilized. The data is collected from I-V curves of each bending cycles; inset: The curved devices with $5 \mathrm{~mm}$ curvature radius. 

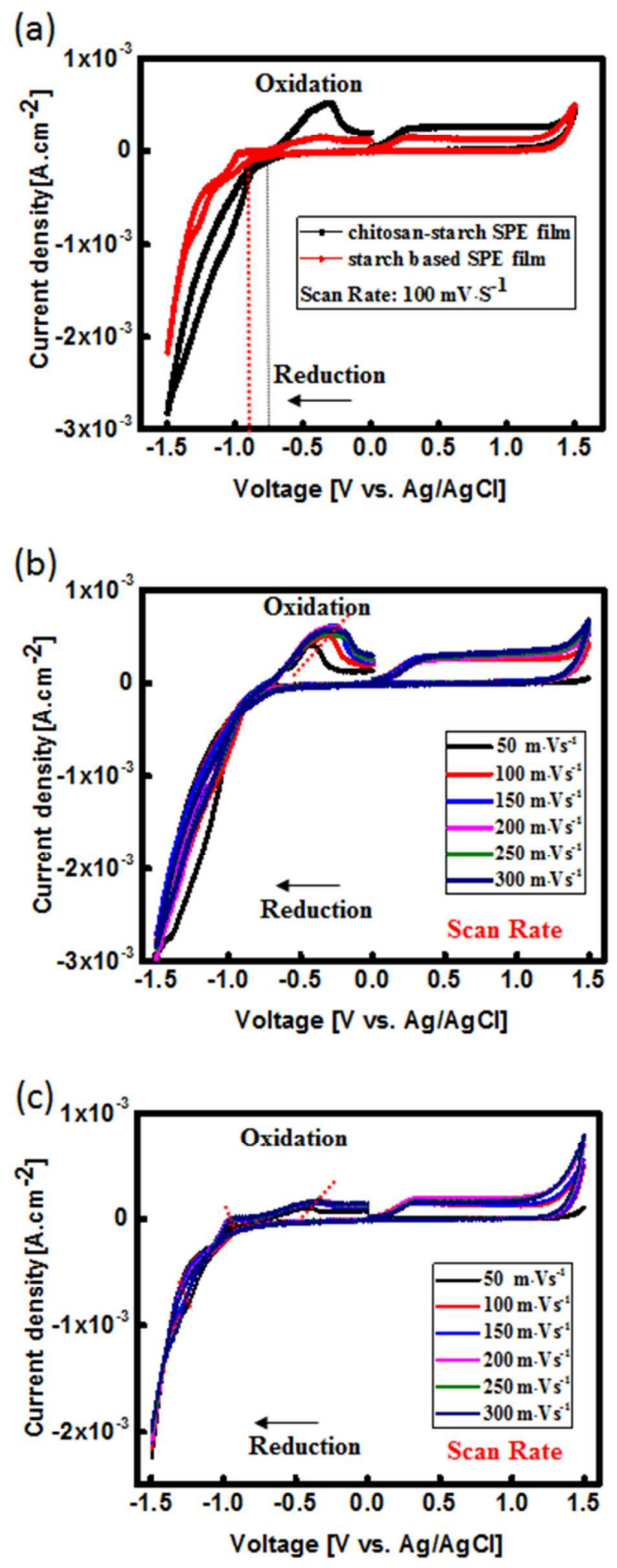

Supporting Figure S5. Electrochemical characterization: (a) Comparison of cyclic voltammogram between starch based and starch-chitosan-based SPE thin film, cyclic voltammetry of (b) starch based and (c) starch-chitosan based SPE with different scanning rates from 50 to $300 \mathrm{mV} \cdot \mathrm{S}^{-1}$. 
(a)

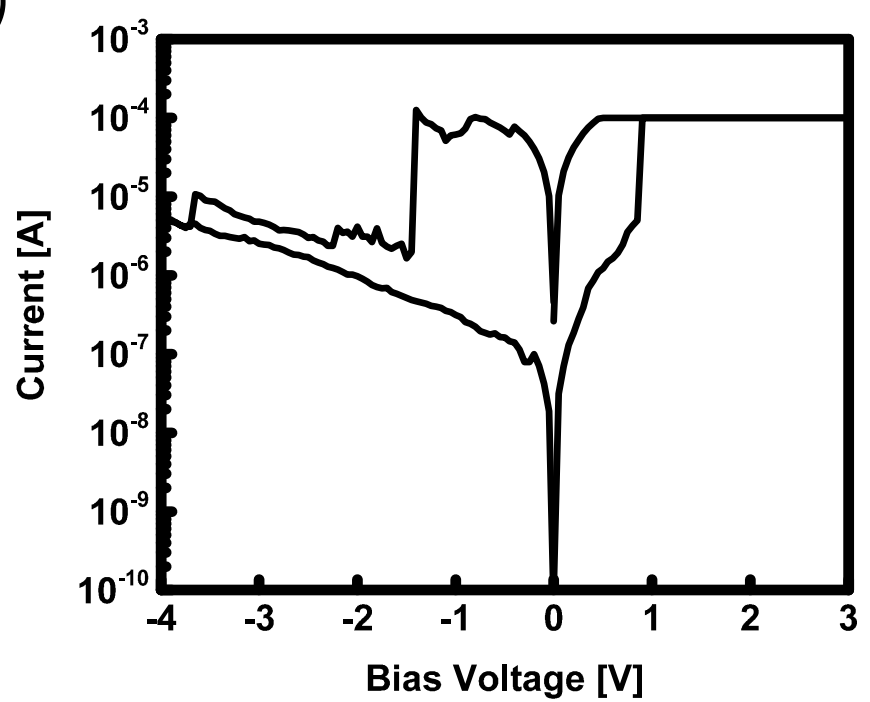

(b)

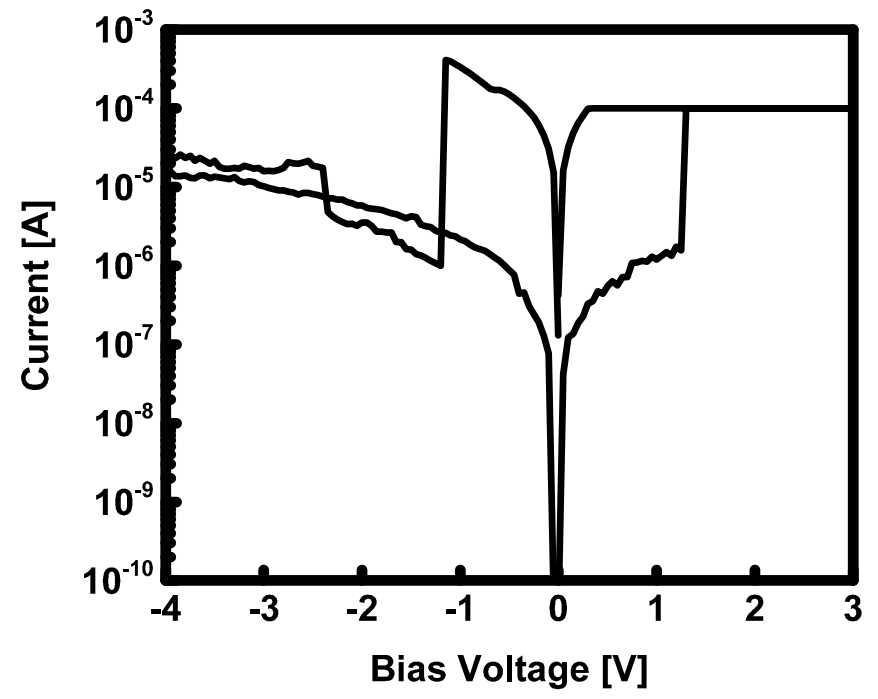

Supporting Figure S6. RS properties of the starch-based device with (a) Al/starch/ITO/PET and (b) Ag/starch/ITO/PET structures. 
(a)
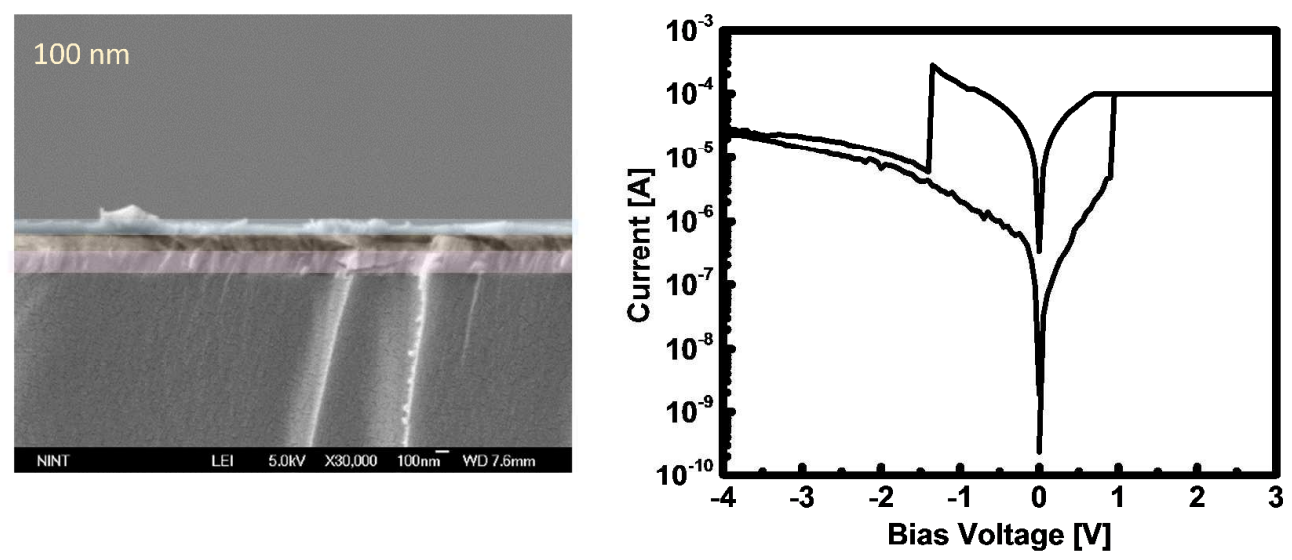

(b)
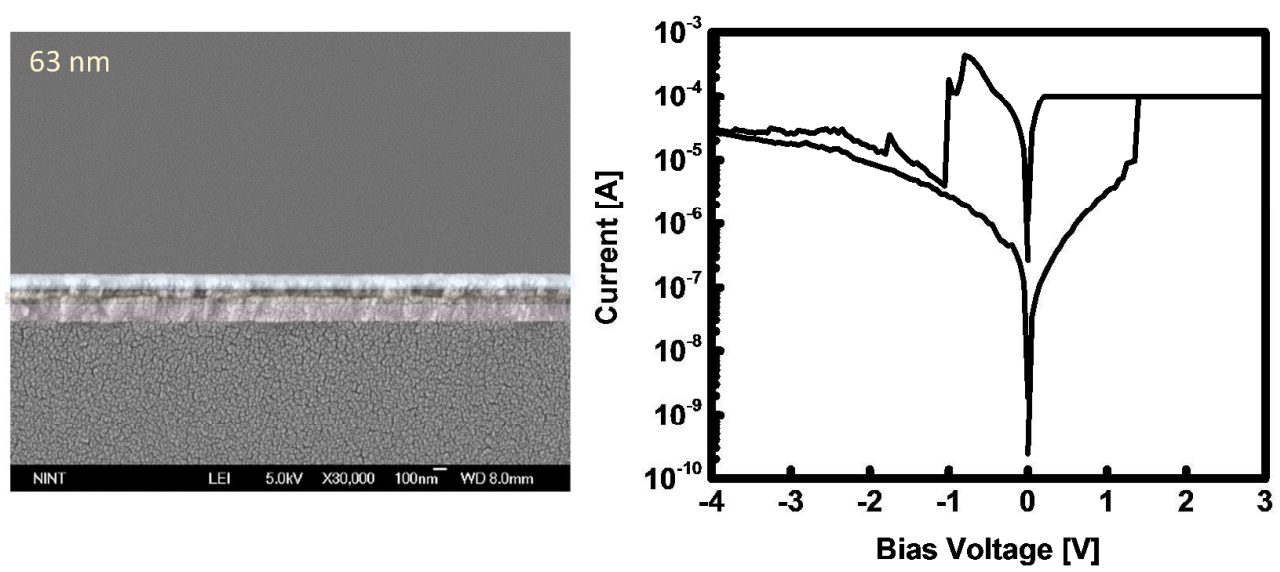

(c)
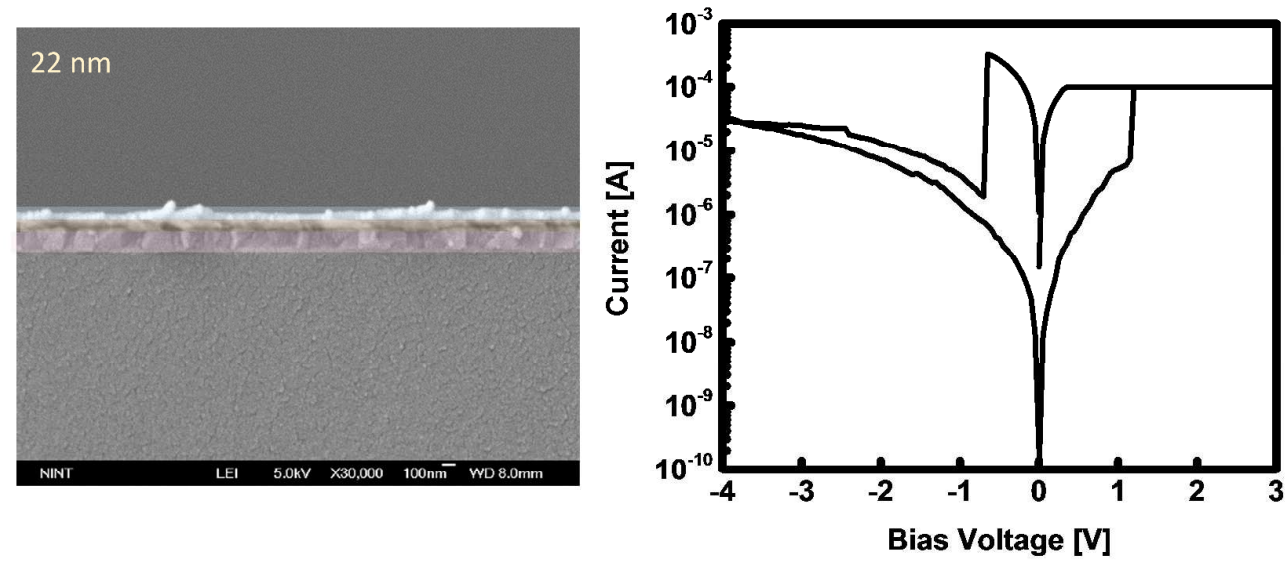

Supporting Figure S7-a. Thickness dependence resistive switching (RS) characteristics of (a-c) starch-based devices; Right-side graphs indicate the RS properties while the left-side figures show the SEM images of the bio-ReRAMs. 
(d)
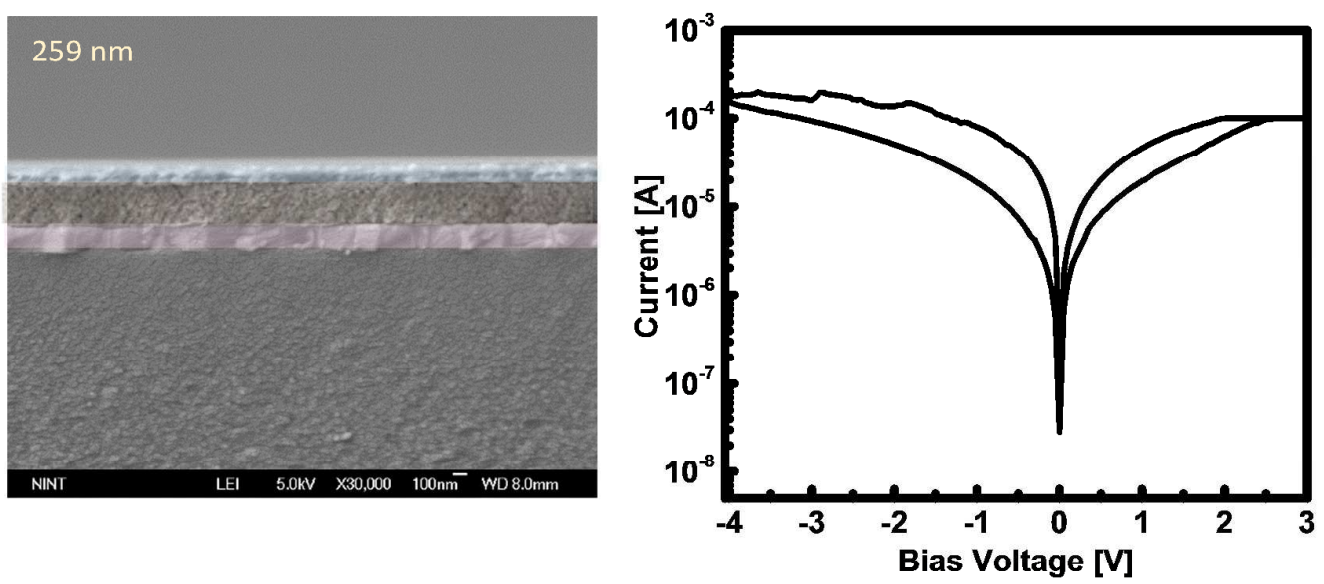

(e)
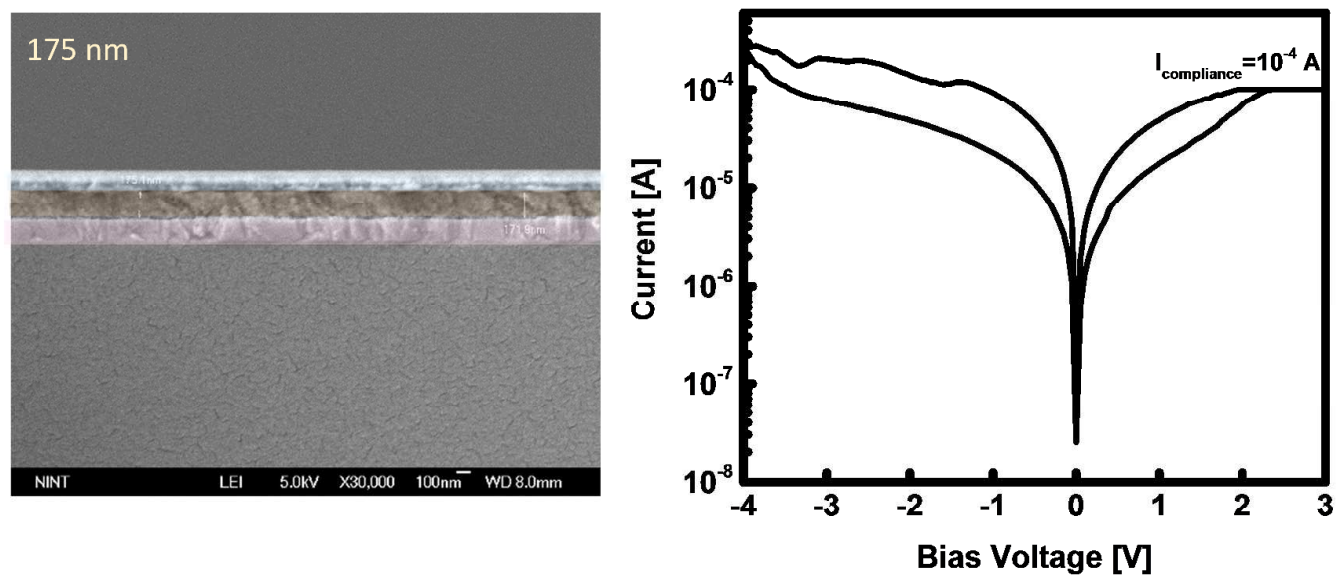

(f)
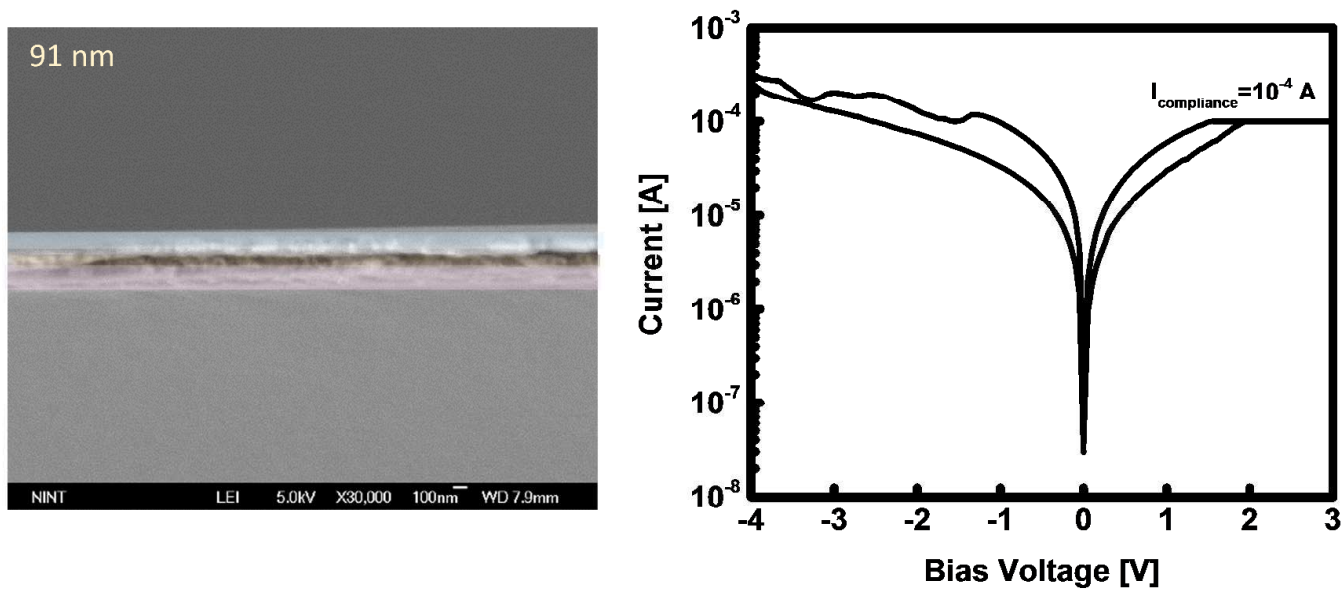

Supporting Figure S7-b. Thickness dependence resistive switching (RS) characteristics of (d-f) starch-chitosan based devices; Right-side graphs indicate the RS properties while the left-side figures show the SEM images of the bio-ReRAMs. 


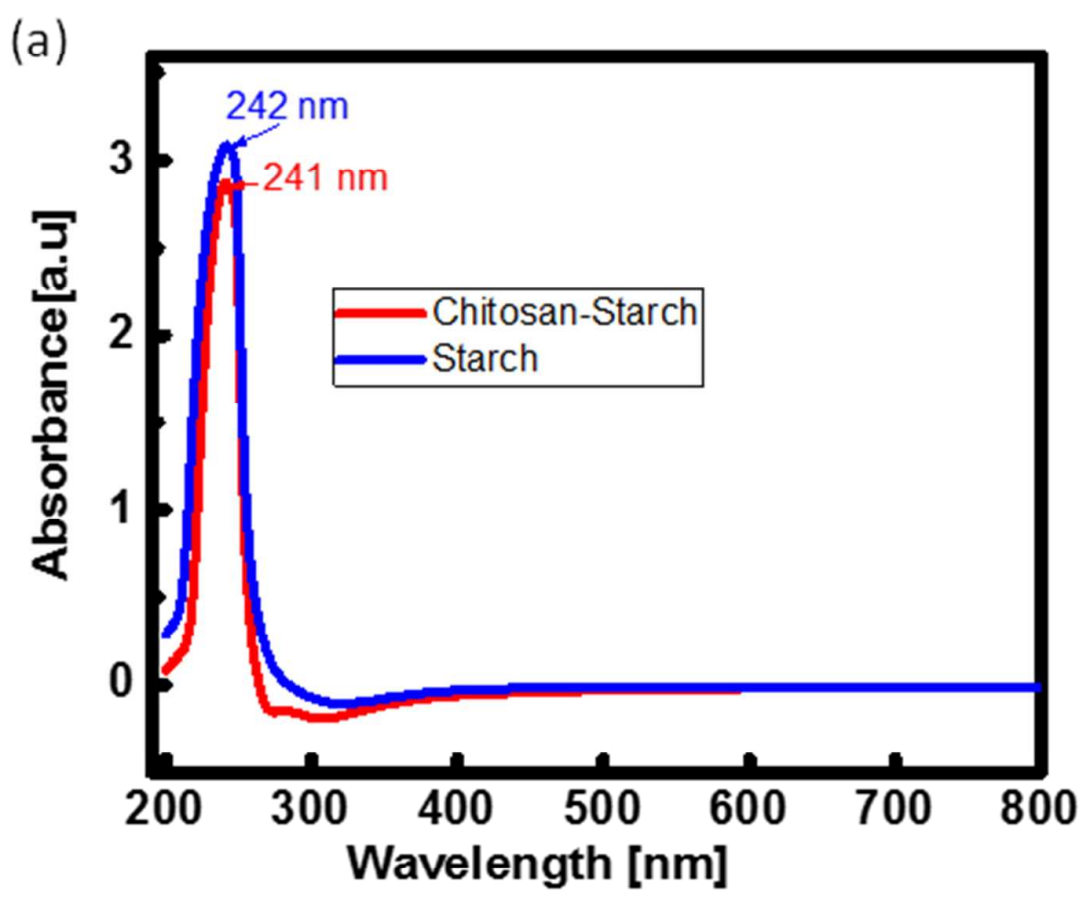

(b)

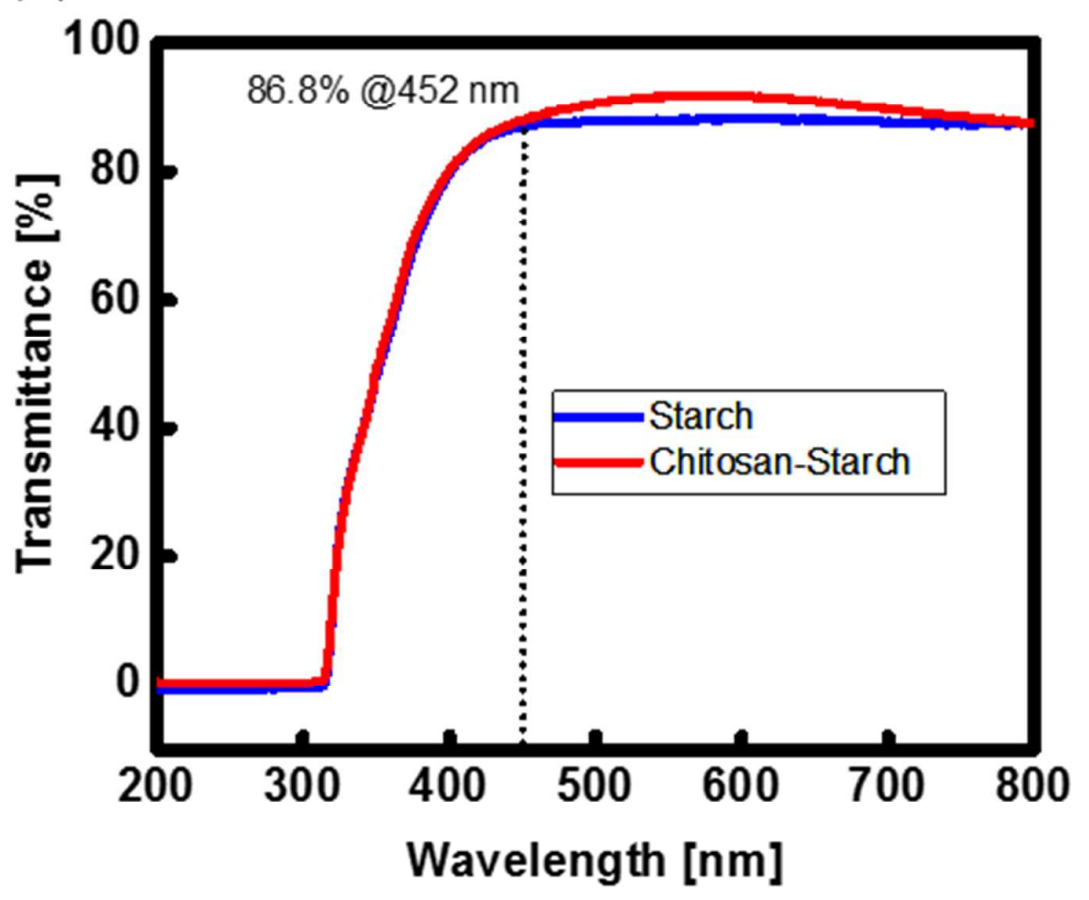

Supporting Figure S8. (a) UV-visible absorption spectra of starch (blue line), and chitosanstarch solution (red line). (b) Transmittance of starch (blue line) and chitosan-starch (red line) on ITO coated PET substrate. 